\title{
Exponential parameterization of neutrino mixing matrix with account of CP-violation data
}

\author{
Konstantin Zhukovsky ${ }^{\mathrm{a}}$, Francisco Melazzini ${ }^{\mathrm{b}}$ \\ Department of Theoretical Physics, Faculty of Physics, M.V. Lomonosov Moscow State University, Moscow 119991, Russia
}

Received: 1 July 2016 / Accepted: 1 August 2016 / Published online: 17 August 2016

(C) The Author(s) 2016. This article is published with open access at Springerlink.com

\begin{abstract}
The exponential parameterization of the Pontecorvo-Maki-Nakagawa-Sakata mixing matrix for neutrinos is discussed. The exponential form allows easy factorization and separate analysis of the CP-violating and Majorana terms. Based upon the recent experimental data on the neutrino mixing, the values for the exponential parameterization matrix for neutrinos are determined. The matrix entries for the pure rotational part in charge of the mixing without $\mathrm{CP}$ violation are derived. The complementarity hypothesis for quarks and neutrinos is demonstrated. A comparison of the results based on most recent and on old data is presented. The $\mathrm{CP}$-violating parameter value is estimated, based on the so far imprecise experimental indications, regarding $\mathrm{CP}$ violation for neutrinos. The unitarity of the exponential parameterization and the CP-violating term transform is confirmed. The transform of the neutrino mass state vector by the exponential matrix with account of $\mathrm{CP}$ violation is shown.
\end{abstract}

\section{Introduction}

One of the paramount achievements of physics of the 20th century was certainly the formulation of the Standard Model [1-3], which unifies the description of electromagnetic and weak interactions in one theory. An important role in the Standard Model is played by neutrinos. In the framework of the Standard Model neutrinos may have three flavors, matching three charged leptons, with which they interact by means of weak interaction. The proper states form full normalized orthogonal basis. Originally the Standard Model assumed massless neutrinos; later it was adapted to incorporate their mass. The existence of the mass of the neutrinos means the existence of at least three massive neutrino states $\boldsymbol{v}_{\mathbf{1}}, \boldsymbol{v}_{\mathbf{2}}, \boldsymbol{v}_{\mathbf{3}}$, and, also, it means the existence of the neutrino oscillations [4], i.e., neutrino flavors change while they

\footnotetext{
a e-mail: zhukovsk@physics.msu.ru

be-mail: francisco.melazzini@physics.msu.ru
}

are propagating. Evidence for that was found in experiments for mixing of solar neutrinos [5], atmospheric neutrinos [6], and reactor neutrinos [7]. The phenomenon of neutrino mixing was predicted by Pontecorvo [8-10]. The 2015 Nobel Prize in physics recognized the discovery of neutrino oscillations. The transforms from the mass state basis to the flavor state basis and vice versa is performed with the help of the Pontecorvo-Maki-Nakagawa-Sakata (PMNS) matrix. This transform can be considered similarly to the quark mixing by the CKM matrix. The familiar neutrino states, $\boldsymbol{v}_{\boldsymbol{e}}, \boldsymbol{v}_{\boldsymbol{\mu}}, \boldsymbol{v}_{\boldsymbol{\tau}}$, are linear combinations of neutrinos with different masses, $v_{1}, v_{2}, v_{3}$ :

$\left|v_{\alpha}\right\rangle=\sum_{i=1,2,3} \mathbf{V}^{*}$ PMNS $_{\alpha i}\left|v_{i}\right\rangle, \quad \mathbf{V}_{\mathrm{PMNS}_{\alpha i}} \equiv\left\langle v_{\alpha} \mid v_{i}\right\rangle$,

where $\mathbf{V}_{\boldsymbol{P} M \boldsymbol{N}}$ is the unitary PMNS mixing matrix [11]. Note the analogy with the mixing of the low elements of left components of the quark spinors, $\left(\begin{array}{l}u \\ d^{\prime}\end{array}\right)_{L},\left(\begin{array}{l}c \\ s^{\prime}\end{array}\right)_{L},\left(\begin{array}{l}t \\ b^{\prime}\end{array}\right)_{L}$. Lepton mixing presumes that a charged $\mathbf{W}$-boson can couple to any mass state of charged leptons $(\boldsymbol{e}, \boldsymbol{\mu}, \boldsymbol{\tau})$ with any mass state of neutrino. For example, $\mathbf{V}_{\alpha i}$ would be the amplitude of the boson $\mathbf{W}^{+}$decay in a pair of lepton type $\boldsymbol{\alpha}$ and neutrino type $i$ and, hence, the production of the lepton $\alpha$ and neutrino state $\alpha$ implies that all neutrino mass states participate in it in a superposition. In the following we do not consider a sterile neutrino, [12-14], which does not interact with Wand $\mathbf{Z}$-bosons. Thus, we end up with a unitary $3 \times 3$ mixing matrix $\mathbf{V}$, factorized by the matrix:

$\mathbf{V}_{\mathrm{PMNS}}=\mathbf{V P}_{M j r}$

where

$\mathbf{V}=\left(\begin{array}{lll}c_{12} c_{13} & s_{12} c_{13} & s_{13} e^{-i \delta} \\ -s_{12} c_{23}-c_{12} s_{23} s_{13} e^{i \delta} & c_{12} c_{23}-s_{12} s_{23} s_{13} e^{i \delta} & s_{23} c_{13} \\ s_{12} s_{23}-c_{12} c_{23} s_{13} e^{i \delta} & -c_{12} s_{23}-s_{12} c_{23} s_{13} e^{i \delta} & c_{23} c_{13}\end{array}\right)$, 
$\mathbf{P}_{M j r}=\operatorname{diag}\left(e^{i \alpha_{1} / 2}, e^{i \alpha_{2} / 2}, 1\right)$,

$c_{i j}=\cos \theta_{i j}, s_{i j}=\sin \theta_{i j}, i, j=1,2,3$, and $\mathbf{P}_{M j r}=\operatorname{diag}$ $\left(e^{i \alpha_{1} / 2}, e^{i \alpha_{2} / 2}, 1\right)$ describes the possible Majoranas nature of neutrinos by the phases $\alpha_{1}$ and $\alpha_{2}$. If $\alpha_{1,2} \neq 0$, then the neutrinos are the Majorana particles, i.e. they are identical to their antiparticles; the phases $\alpha_{1}, \alpha_{2}$ play a role in the processes, which do not preserve the lepton number. The role of the matrix $\mathbf{V}$ in the parameterization (3) is similar to the one that the CKM matrix plays in quark mixing [15,17-20]. The PMNS matrix is fully determined by four parameters: three mixing angles $\theta_{12}, \theta_{23}, \theta_{13}$, and the phase $\delta$, in charge of the $\mathrm{CP}$ violation description [15]. The experimental values of the mixing angles are relatively well determined $[15,16,21-$ 24]:

$\theta_{12} \cong 33.36^{\circ} \pm 0.8^{\circ}$,

$\theta_{23} \cong 40.0^{\circ} \pm 2^{\circ}$,

$\theta_{13} \cong 8.66^{\circ} \pm 0.45^{\circ}$.

Contrary to quark mixing angles, these are not small and the expansion in series of the only small parameter is not possible. Thus, there is no small parameter, like $\lambda=\sin$ $\theta_{\text {Cabibbo }} \approx 0.22$ [25], for neutrino mixing. Experimentally determined absolute values for the elements of the PMNS matrix read as follows [15]:

$$
|\mathbf{V}|=\left(\begin{array}{lll}
0.82 & 0.54 & 0.15 \\
0.35 & 0.70 & 0.62 \\
0.44 & 0.45 & 0.77
\end{array}\right)
$$

Moreover, there are indications that the $\mathrm{CP}$-violating phase may have a non-zero value; moreover, very approximately it is supposed to be as large as $\delta \approx 300^{\circ}$ (see [26,27]).

\section{Exponential mixing matrix}

The exponential parameterization for the neutrino mixing matrix was outlined in [28] and then in [29]; it is constructed similarly to that for quarks [30,31]. Unitarity of the exponential mixing matrix $\mathbf{V}=\exp \mathbf{A}$ is guaranteed by the anti-Hermitian form of the exponent $\mathbf{A}=$ $\left(\begin{array}{lll}0 & \lambda_{1} & \lambda_{3} e^{i \delta_{C P}} \\ -\lambda_{1} & 0 & -\lambda_{2} \\ -\lambda_{3} e^{-i \delta_{C P}} & \lambda_{2} & 0\end{array}\right)$ (see [33]), which depends on the mixing parameters $\lambda_{i}$, and on the CP-violation phase $\delta_{\mathrm{CP}}$. Note that for $\delta_{\mathrm{CP}}=2 \pi n$ we obtain simply a rotation matrix around the axis in space [31]. The matrix also becomes real for $\delta_{\mathrm{CP}}=\pi(2 n+1)$. The most important advantage of the exponential parameterization for the mixing matrix with respect to the commonly known standard parameterization is that the exponential parameterization allows easy separation of the contributions of the rotation part, the $\mathrm{CP}$ violation and possible other terms in stand alone factors. This separation can be made in a variety of modes, of which we omit the details here; proper discussion was presented, for example, in $[31,32]$. The following unitary parameterization was proposed in [29]:

$\tilde{\mathbf{V}}=\mathbf{P}_{\mathbf{R o t}} \mathbf{P}_{\mathbf{C P}} \mathbf{P}_{\mathbf{M j r}}$,

where the rotation part is given by the real exponential matrix

$\mathbf{P}_{\mathbf{R o t}}=e^{\mathbf{A}_{\text {Rot }}}=\exp \left(\begin{array}{lll}0 & \lambda & \mu \\ -\lambda & 0 & -v \\ -\mu & v & 0\end{array}\right)$,

the $\mathrm{CP}$ violation is accounted for by

$\mathbf{P}_{\mathrm{CP}}=e^{\mathbf{A}_{\mathrm{CP}}}$,

and it contains an imaginary component,

$\mathbf{A}_{\mathbf{C P}}=\left(\begin{array}{lll}0 & 0 & \mu\left(-1+e^{i \delta_{C P}}\right) \\ 0 & 0 & 0 \\ \mu\left(1-e^{-i \delta_{C P}}\right) & 0 & 0\end{array}\right)$,

and the Majorana part,

$\mathbf{P}_{M j r}=e^{\mathbf{A}_{M j r}}$,

depends on the Majorana phases in the exponential:

$\mathbf{A}_{\mathbf{M j r}}=i\left(\begin{array}{lll}\alpha_{1} / 2 & 0 & 0 \\ 0 & \alpha_{2} / 2 & 0 \\ 0 & 0 & 0\end{array}\right)$

The details of the splitting between CP-conserving and CPviolating terms in the above parameterization can be found in [29] (also compare with [31,32]). The values of the Majorana phases $\alpha_{1}$ and $\alpha_{2}$ are at present undetermined; the value of the CP-violating phase $\delta_{\mathrm{CP}}$ can be figured from the existing experimental indications and estimations (see, for example, $[26,27])$.

The rotation matrix can be conveniently presented in the form of the rotation in the angle $\Phi$ around the axis, given by the vector $\overrightarrow{\mathbf{n}}=\left(n_{x}, n_{y}, n_{z}\right)$ :

$\begin{aligned} \mathbf{M}(\overrightarrow{\mathbf{n}}, \Phi)=e^{\Phi} \mathbf{N} & =\left(\begin{array}{lll}M_{x x} & M_{x y} & M_{x z} \\ M_{y x} & M_{y y} & M_{y z} \\ M_{z x} & M_{z y} & M_{z z}\end{array}\right), \\ \mathbf{N} & =\left(\begin{array}{lll}0 & -n_{z} & n_{y} \\ n_{z} & 0 & -n_{x} \\ -n_{y} & n_{x} & 0\end{array}\right) .\end{aligned}$

In this form it represents a three-dimensional rotation generator. Then (15) links the entries of the rotational matrix (10) in the exponential parameterization (9) with the rotation angle 


$$
\Phi= \pm \sqrt{\lambda^{2}+\mu^{2}+v^{2}}
$$

around the axis $\overrightarrow{\mathbf{n}}=\left(n_{x}, n_{y}, n_{z}\right)$ with the following coordinates:

$n_{x}=\frac{v}{\Phi}, \quad n_{y}=\frac{\mu}{\Phi}, \quad n_{z}=-\frac{\lambda}{\Phi}$.

Thus, Eqs. (17)-(16) relate the elements of the exponential parameterization with the single axis-rotation angle matrix components. Note that the angles of the rotation in the standard parameterization matrix, (3) $c_{i j}=\cos \theta_{i j}$ and $s_{i j}=$ $\sin \theta_{i j}$, are different from those in our parameterization (9). Omitting the Majorana part, the exponential parameterization (9) reads as follows:

$$
\begin{aligned}
\tilde{\mathbf{V}} & =\mathbf{M P}_{\mathbf{C P}} \\
& =\left(\begin{array}{lll}
M_{x x} \cos 2 \Omega+\omega_{-} M_{x z} \sin 2 \Omega & M_{x y} & M_{x z} \cos 2 \Omega+\omega_{+} M_{x x} \sin 2 \Omega \\
M_{y x} \cos 2 \Omega+\omega_{-} M_{y z} \sin 2 \Omega & M_{y y} & M_{y z} \cos 2 \Omega+\omega_{+} M_{y x} \sin 2 \Omega \\
M_{z x} \cos 2 \Omega+\omega_{-} M_{z z} \sin 2 \Omega & M_{z y} & M_{z z} \cos 2 \Omega+\omega_{+} M_{z x} \sin 2 \Omega
\end{array}\right),
\end{aligned}
$$

where

$\Omega=\mu \sin \frac{\delta_{\mathrm{CP}}}{2}, \quad \omega_{ \pm}=e^{\frac{i}{2}\left(\pi \pm \delta_{C P}\right)}$.

The values of the entries of the rotation matrix $M_{i j}$ can be derived from the following tensor identity (see [34]):

$$
\begin{aligned}
M_{i j}= & (1-\cos \Phi) n_{i} n_{j} \\
& +\delta_{i j} \cos \Phi-\varepsilon_{i j k} n_{k} \sin \Phi, \quad i, j, k=x, y, z,
\end{aligned}
$$

where we denote $\delta_{i j}$ the Kronecker symbol, $\varepsilon_{i j k}$ is the LeviCivita symbol, $n_{i}$ are the components of the vector $\overrightarrow{\mathbf{n}}=$ $\left(n_{x}, n_{y}, n_{z}\right)$ and $\Phi$ is the rotation angle. The expressions, relating the entries of the standard parameterization $c_{i j}$ and $s_{i j}$ with $\overrightarrow{\mathbf{n}}$ and $\Phi$, can be derived from (18), (19), (20), and (3), but they are very cumbersome and we omit them for brevity. From the experimental data [26] by using matrix equations and expansions we obtain for the rotation vector in 3D space the following coordinates:

$n_{x} \cong 0.702, \quad n_{y} \cong 0.394, \quad n_{z} \cong 0.593$,

and for the rotation angle around this axis we obtain

$\Phi \cong 49.8^{\circ}$.

The precision of the above values is determined by the errors in the experimental data evaluation and is about of $5 \%$. From (15)-(17) we obtain the following values for the entries of the rotation matrix (15) in the exponential parameterization:

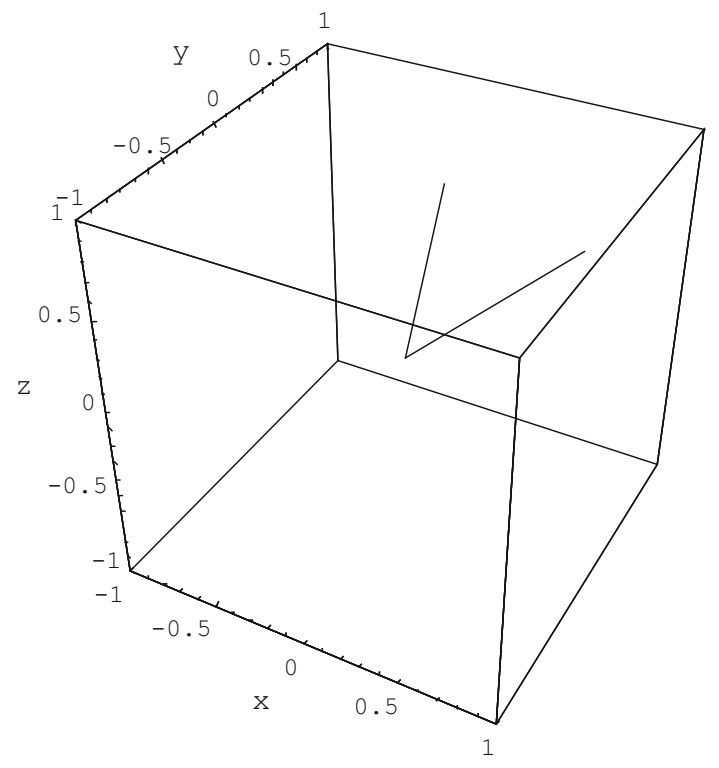

Fig. 1 The axes of rotation for quarks and neutrinos form the angle of $\approx 45^{\circ}$

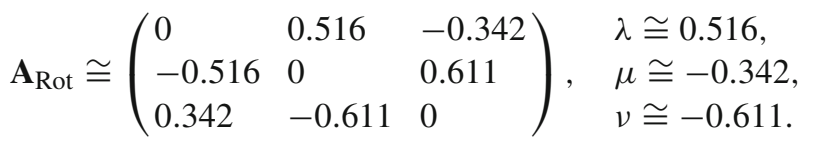

This result is in agreement with the experimental data and with the respective standard parameterization in the PMNS matrix (2). Moreover, based upon the values of the mixing angles for quarks $\theta_{Q 12}=13.14^{\circ}, \theta_{Q 23}=2.43^{\circ}, \theta_{Q 13}=$ $0.23^{\circ}$, we determine the direction of the rotation vector in space (17) for the exponential parameterization for quarks as follows:

$\overrightarrow{\mathbf{n}}_{\text {quark }}=(0.1829,0.0206,0.9831)$.

Now, upon the comparison with the above determined coordinates of the rotation vector for neutrinos

$\overrightarrow{\mathbf{n}}_{\text {neutrino }}=(0.7021,0.3936,0.5934)$,

we note that $\overrightarrow{\mathbf{n}}_{\text {quark }}$ and $\overrightarrow{\mathbf{n}}_{\text {neutrino }}$ constitute the angle of $\approx 44^{\circ}$, as demonstrated in Fig. 1.

This fact is interesting itself and it is the demonstration of the so-called hypothesis of complementarity for neutrinos and quarks $[35,36]$, according to which the rotation axes for quarks and neutrinos form the $45^{\circ}$ angle; however, this last statement is rather an observation since it does not have solid theoretical foundation and there are no physical reasons. Note that the obtained value of $44^{\circ}$ differs from $45^{\circ}$ by $\approx 2 \%$, which is within the margin of errors of the original experimental data sets, which determines the entries of the exponential mixing matrix and the rotation vectors directions. 


\section{Exponential parameterization and $\mathrm{CP}$ violation}

Now let us take advantage of the possibility, given by the exponential parameterization, which allows us to factorize separately the contributions of the rotation, the $\mathrm{CP}$-violation, and the Majorana term. We can write the matrix product $\mathbf{P}_{\mathbf{C P}} \mathbf{P}_{\mathbf{M j r}}$ in the following form, which reminds one of the rotation by the angle $2 \Omega$ with the proper weights for each entry:

$$
\begin{aligned}
& \mathbf{V}_{\mathbf{M C P}}=\mathbf{P}_{\mathbf{C P}} \mathbf{P}_{\mathbf{M j r}}=\left(\begin{array}{lll}
e^{i \frac{\alpha_{1}}{2}} \cos 2 \Omega & 0 & \omega_{+} \sin 2 \Omega \\
0 & e^{i \frac{\alpha_{2}}{2}} & 0 \\
\omega_{-} e^{i \frac{\alpha_{1}}{2}} \sin 2 \Omega & 0 & \cos 2 \Omega
\end{array}\right) \\
& =\left(\begin{array}{lll}
e^{i \frac{\alpha_{1}}{2}} \cos \left(2 \mu \sin \frac{\delta_{C P}}{2}\right) & 0 & e^{\frac{i}{2}\left(\pi+\delta_{C P}\right)} \sin \left(2 \mu \sin \frac{\delta_{C P}}{2}\right) \\
0 & e^{i \frac{\alpha_{2}}{2}} & 0 \\
e^{\frac{i}{2}\left(\pi-\delta_{C P}+\alpha_{1}\right)} \sin \left(2 \mu \sin \frac{\delta_{C P}}{2}\right) & 0 & \cos \left(2 \mu \sin \frac{\delta_{C P}}{2}\right)
\end{array}\right) .
\end{aligned}
$$

Omitting the Majorana part, the complexity due to the $\mathrm{CP}$ violation vanishes if either $\Omega= \pm \pi n / 2$, or if $\omega_{ \pm} \in$ Reals. The value of $\Omega$ is limited by $\mu$, which is small, and the above expression (26) becomes real in the case of $\Omega=0$, which, moreover, returns the unity matrix $\mathbf{P}_{\mathbf{C P}}=\mathbf{I}$ for $\delta_{\mathrm{CP}}= \pm 2 \pi n$. The absolute values of the entries of the CP-violating matrix are at their maximum for $\delta_{\mathrm{CP}}=\pi \pm 2 \pi n$, but then the whole $\mathbf{P}_{\mathbf{C P}}$ matrix becomes real and the $\mathrm{CP}$ violation in fact vanishes. The major contribution of the complex term due to the $\mathrm{CP}$ violation is achieved for $\delta_{\mathrm{CP}}=\pi / 2 \pm \pi n$. All of the above is in complete analogy with the behavior of the CP-violating phase $\delta$ in the standard parameterization. Based upon the present knowledge of the $\mathrm{CP}$ violation for neutrinos and on experimental indications and estimations $[26,27]$ and accounting for the above discussion, the CPviolating phase in the exponential parameterization has the value $\delta_{\mathrm{CP}} \cong-60^{\circ}$ identical to $\delta \approx 300^{\circ}$. Then we obtain

$$
\left.\omega_{ \pm}\right|_{\delta_{\mathrm{CP}}=-60^{\circ}} e^{\frac{i \pi}{2}\left(1 \mp \frac{1}{3}\right)} \cong \pm 0.5+0.866 i \text {. }
$$

Note that the accuracy of the existing indications with regard to the $\mathrm{CP}$ violation is rather low $(+60-120 \%)$ and thus the above values are also approximate. The parameter $\Omega$ is
$\Omega=\mu \sin \frac{\delta \mathrm{CP}}{2}=\mu \sin \left(-30^{\circ}\right)=-\mu / 2=0.171$,

$\cos 2 \Omega=\cos \mu \cong 0.942, \quad \sin 2 \Omega=-\sin \mu \cong 0.335$.

Then with account of the Majorana phases and of $\delta_{\mathrm{CP}} \cong$ $-60^{\circ}$, we obtain the following matrix:

$$
\begin{aligned}
& \mathbf{V}_{\mathbf{M C P}}=\mathbf{P}_{\mathbf{C P}} \mathbf{P}_{\mathbf{M j r}}=\left(\begin{array}{lll}
e^{i \frac{\alpha_{1}}{2}} 0.942 & 0 & 0.335 \omega_{+} \\
0 & e^{i \frac{\alpha_{2}}{2}} & 0 \\
0.335 e^{i \frac{\alpha_{1}}{2}} \omega_{-} & 0 & 0.942
\end{array}\right) \\
& \cong\left(\begin{array}{lll}
0.942 e^{i \frac{\alpha_{1}}{2}} & 0 & 0.168+0.290 i \\
0 & e^{i \frac{\alpha_{2}}{2}} & 0 \\
e^{i \frac{\alpha_{1}}{2}}(-0.168+0.290 i) & 0 & 0.942
\end{array}\right) .
\end{aligned}
$$

For Dirac neutrinos at the extremities of the range of the CPviolating phase for $\delta_{\mathrm{CP}}=0^{\circ}$ we get the unity matrix $\mathbf{P}_{\mathbf{C P}}=$ I, while for $\delta_{\mathrm{CP}} \cong-180^{\circ}:\left.\omega_{ \pm}\right|_{\delta_{\mathrm{CP}}=-180^{\circ}}=e^{\frac{i \pi}{2}(1 \mp 1)}= \pm 1$ and the real mixing term

$\mathbf{P}_{\mathbf{C P}} \equiv \mathbf{V}_{\mathbf{M C P}}=\left(\begin{array}{lll}0.775 & 0 & 0.632 \\ 0 & 1 & 0 \\ -0.632 & 0 & 0.775\end{array}\right)$.

The Majorana phases interplay with the CP-phase only in the entry $(3,1)$ in the factor $e^{i \frac{\alpha_{1}}{2}} \omega_{-}$. Otherwise, the Majorana phases just bring about more complexity in the result. For non-Majorana, but Dirac particles, the matrix obtained above, (29), represents just a slight deviation from the unitary matrix I:

$\mathbf{P}_{\mathbf{C P}} \equiv \mathbf{V}_{\mathbf{M C P}} \cong\left(\begin{array}{lll}1 & 0 & 0.168+0.290 i \\ 0 & 1 & 0 \\ -0.168+0.290 i & 0 & 1\end{array}\right)$.

It is now evident that for non-Majorana particles the CPviolating term (29) is the mixing matrix for two lepton generations: electron, tauon, and proper neutrinos. Employing the generating functions for the Bessel functions $\cos (x \sin \alpha)=\sum_{n=-\infty}^{\infty} J_{n}(x) \cos n \alpha$ and $\sin (x \sin \alpha)=$ $\sum_{n=-\infty}^{\infty} J_{n}(x) \sin n \alpha$, we easily derive the following expression for the general form of the matrix $\mathbf{V}_{\mathbf{M C P}}$, in which the contributions of the CP-violating phase $\delta_{C P}$ and of the rotation matrix parameter $\mu$ are separated:

$\mathbf{V}_{\mathbf{M C P}}=\left(\begin{array}{lll}e^{i \frac{\alpha_{1}}{2}} \sum_{n=-\infty}^{\infty} J_{n}(2 \mu) \cos \left(\frac{n \delta_{C P}}{2}\right) & 0 & \omega_{+} \sum_{n=-\infty}^{\infty} J_{n}(2 \mu) \sin \left(\frac{n \delta_{C P}}{2}\right) \\ 0 & e^{i \frac{\alpha_{2}}{2}} & 0 \\ \omega-e^{i \frac{\alpha_{1}}{2}} \sum_{n=-\infty}^{\infty} J_{n}(2 \mu) \sin \left(\frac{n \delta_{C P}}{2}\right) & 0 & \sum_{n=-\infty}^{\infty} J_{n}(2 \mu) \cos \left(\frac{n \delta_{C P}}{2}\right)\end{array}\right)$.

not very sensitive to the exact value of the angle $\delta_{\mathrm{CP}}$ and its absolute value varies from 0 to the maximum of $|\mu|=0.342$ for $\delta_{\mathrm{CP}}=\pi \pm 2 \pi n$. Note that in any case the value of $\Omega$ is small and $\cos \Omega \cong 1$. For $\delta_{\mathrm{CP}}=-60^{\circ}$ we obtain
The above result (32) is valid for arbitrary values of $\delta_{\mathrm{CP}}$, $\alpha_{1}, \alpha_{2}$. For $\alpha_{1}=0$, (32) reduces to the symmetric form. With account of the obtained values of the entries of the exponential parameterization of the $\mathrm{CP}$-violating term and for non-zero Majorana phases $\alpha_{1}, \alpha_{2}$, the vector of the mixed 
neutrino state is transformed as follows:

$$
\begin{aligned}
\left|v_{\alpha}\right\rangle & =\mathbf{M}\left(\begin{array}{l}
0.942 e^{-\frac{i}{2} \alpha_{1}}\left|v_{1}\right\rangle+0.335 e^{-\frac{\pi}{3} i}\left|v_{3}\right\rangle \\
e^{-i \frac{\alpha_{2}}{2}}\left|\nu_{2}\right\rangle \\
0.335 e^{-i\left(\frac{\alpha_{1}}{2}+\frac{2 \pi}{3}\right)}\left|\nu_{1}\right\rangle+0.942\left|\nu_{3}\right\rangle
\end{array}\right) \\
& \cong \mathbf{M}\left(\begin{array}{l}
e^{-\frac{i}{2} \alpha_{1}}\left|\nu_{1}\right\rangle+(0.168-0.290 i)\left|\nu_{3}\right\rangle \\
e^{-i \frac{\alpha_{2}}{2}}\left|\nu_{2}\right\rangle \\
-(0.168+0.290 i) e^{-\frac{i}{2} \alpha_{1}}\left|v_{1}\right\rangle+\left|v_{3}\right\rangle
\end{array}\right) .
\end{aligned}
$$

We underline that the above transform by $\mathbf{V}_{\mathbf{M C P}}$, as well as the transform by the purely rotational part $\mathbf{P}_{\mathbf{R o t}}$, is unitary. It can be verified directly with the help of the Hermiteconjugated matrix:

$\mathrm{V}_{\mathrm{MCP}}^{-1} \cdot \mathrm{V}_{\mathrm{MCP}}=\mathrm{V}_{\mathrm{MCP}}^{+} \cdot \mathrm{V}_{\mathrm{MCP}}=\mathrm{I}$.

This ensures the unitarity of the whole exponential parameterization (9) of the PMNS mixing matrix:

$\tilde{\mathbf{V}}^{-1} \cdot \tilde{\mathbf{V}}=\tilde{\mathbf{V}}^{+} \cdot \tilde{\mathbf{V}}=\mathbf{I}$.

\section{Conclusions}

The exponential parameterization of the mixing matrix for neutrinos is explored with account of the present experimental data. The proper entries of the exponential mixing matrix are determined; the CP-violating term in the exponential parameterization is estimated. Based upon the accuracy of the experimental data, the range of the values for the parameters of the neutrino mixing matrix is given. Without $\mathrm{CP}$ violation the neutrino mixing represents in fact the geometric rotation in three-dimensional space. In this simple case mixing can be viewed as the rotation by the angle $\Phi$ around the axis in threedimensional space. This interpretation follows straightforwardly from the structure of the exponential mixing matrix. Evidently, there is no mixing for $\Phi=0$, when the mixing matrix without $\mathrm{CP}$ violation reduces to the unit matrix, I. Based upon the recent data, we have obtained the value for the rotation angle $\Phi \cong 49.8^{\circ}$ and the coordinates of the rotation vector $\overrightarrow{\mathbf{n}}=(0.702,0.394,0.593)$. This value of the rotation angle is somewhat smaller than that based upon the tribimaximal parameterization: $\Phi_{\mathrm{TBM}} \cong 56.6^{\circ}$. Moreover, the direction of the rotation vector differs from that of the vector for the TBM matrix: $\overrightarrow{\mathbf{n}}_{\mathrm{TBM}}=(0.7858,0.2235,0.5777)$. The difference in their directions in $3 \mathrm{D}$ space is $11^{\circ}$. Interestingly, the angle between the axes of rotation for quarks and neutrinos remains unchanged and equals $\approx 45^{\circ}$, despite the change of $11^{\circ}$ in the direction of the neutrinos rotation axis, verified in the last 10 years. This demonstrates the hypothesis of complementarity for quarks and neutrinos [35,36].

The exponential parameterization allows factorization of the $\mathrm{CP}$ and the Majorana contributions and is evidence that the CP-term can also be viewed as a sort of rotation with different weights for the matrix entries. We have calculated the entries of the rotational mixing matrix $\lambda \cong 0.516, \mu \cong$ $-0.342, v \cong-0.611$ (see (23)) and we have estimated the entries of the $\mathrm{CP}$-violating matrix in the exponential parameterization (see (29), (31)) from the current indications on $\mathrm{CP}$ violation: $\delta_{\mathrm{CP}} \sim-60^{\circ}$. This value is quite approximate due to uncertain experimental data, regarding $\mathrm{CP}$ violation for neutrinos. We calculated the $\mathrm{CP}$-violating exponential matrix for the extremities of the range of $\delta_{\mathrm{CP}}$ from $0^{\circ}$ to $180^{\circ}$. The rotation angle $2 \Omega$ for the $\mathrm{CP}$-violating matrix is rather small: $\Omega=\mu / 2$. By means of the exponential parameterization one can easily transform the neutrino state vector, distinguishing the CP-violating terms for each type of neutrino. In the case of Dirac neutrinos and if $\delta_{\mathrm{CP}}= \pm 2 \pi n$, we get pure rotation, since $\mathbf{P}_{\mathbf{C P}}=$ I. If $\delta_{\mathrm{CP}}=\pi \pm 2 \pi n$, then we end with the real $\mathbf{P}_{\mathbf{C P}}$ matrix for the $\mathrm{CP}$-violating term, which means the absence of CP violation in this case. For Majorana neutrinos the mass state vector $\boldsymbol{v}_{\mathbf{1}}, \boldsymbol{v}_{\mathbf{2}}, \boldsymbol{v}_{\mathbf{3}}$ is transformed with complex weights, as demonstrated in (33).

We addressed an exponential presentation of the mixing matrix and obtained with its help results and interpretations that can be useful for the treatment and analysis of new experimental data, regarding the neutrino oscillations in currently running experiments as well as in planned experimental projects.

Acknowledgments We would like to thank professor A.V. Borisov from the Moscow State University, Russia, for his useful suggestions, fruitful discussions, and important advice.

Open Access This article is distributed under the terms of the Creative Commons Attribution 4.0 International License (http://creativecomm ons.org/licenses/by/4.0/), which permits unrestricted use, distribution, and reproduction in any medium, provided you give appropriate credit to the original author(s) and the source, provide a link to the Creative Commons license, and indicate if changes were made. Funded by SCOAP $^{3}$.

\section{References}

1. S. Weinberg, Phys. Rev. Lett 19, 1264 (1967)

2. A. Salam, in Elementary Particle Theory, ed. by N. Svartholm, Almquist Forlag AB, (1968)

3. S.L. Glashow, Nucl. Phys. 22, 579 (1961)

4. V. Barger, D. Marfatia, K.L. Whisnant, in The Physics of Neutrinos (Princeton University Press, Princeton, 2012)

5. T. Ataki et al., KamLAND Collaboration. Phys. Rev. Lett. 94, 081801 (2005)

6. Y. Ashie et al., Super-Kamiokande Collaboration. Phys. Rev. D 71, 112005 (2005)

7. M. Apollonio et al., CHOOZ Collaboration. Eur. Phys. J. C 27, 331 (2003)

8. B. Pontecorvo, Zh.Eksp. Teor. Fiz. 33, 549 (1957)

9. B. Pontecorvo, Zh Eksp, Teor. Fiz. 53, 1717 (1967)

10. B. Pontecorvo, Sov. Phys. JETP 26, 984 (1968)

11. Z. Maki, M. Nakagawa, S. Sakata, Prog. Teor. Phys. 28, 870 (1962) 
12. H. Murayama, T. Yanagida, Phys. Lett. B 520, 263 (2001)

13. G. Barenboim et al., JHEP 0210, 001 (2002)

14. M.C. Gonzalez-Garcia, M. Maltoni, T. Schwetz, Phys. Rev. D 68, 053007 (2003)

15. K.A. Olive et al., Particle Data Group. Chin. Phys. C 38, 090001 (2014)

16. H. Minako, K. Yee, O. Naotoshi, T. Tatsu, A simple parameterization of matter effects on neutrino oscillations. (2006). arXiv:hep-ph/0602115

17. L.-L. Chau, W.-Y. Keung, Phys. Rev. Lett. 53, 1802 (1984)

18. H. Harari, M. Leurer, Phys. Lett. B 181, 123 (1986)

19. H. Fritzsch, J. Plankl, Phys. Rev. D 35, 1732 (1987)

20. F.J. Botella, L.-L. Chao, Phys. Lett. B 168, 97 (1986)

21. B. Aharmim et al., SNO Collaboration. Phys. Rev. C 72, 055502 (2005)

22. T. Araki et al., The KamLAND Collaboration. Phys. Rev. Lett. 94, 081801 (2005)

23. Y.Suzuki, presented at the XXII Int Symp. On Lepton and Photon Interactions at High Energies (Lepton-Photon 2005), Uppsala, Sweden, July 2005
24. C. Bemporad, The Chooz Collaboration. Nucl. Phys. Proc. Suppl. 77, 159 (1999)

25. L. Wolfenstein, Phys. Rev. Lett. 51, 1945 (1983)

26. S.F. King. (2015). arXiv:1510.02091v1 [hep-ph]

27. E. Ma (2015). arXiv:1510.02501v1 [hep-ph]

28. A. Strumia, F. Vissani, "Neutrino masses and mixings and..." (2006). arXiv:hep-ph/0606054

29. G. Datolli, K.V. Zhukovsky, Eur. Phys. J. C 55, 547-552 (2008)

30. G. Dattoli, K. Zhukovsky, Eur. Phys. J. C 50, 817-821 (2007)

31. G. Dattoli, K. Zhukovsky, Eur. Phys. J. C 52(3), 591-595 (2007)

32. G. Dattoli, K.V. Zhukovsky, Phys. At. Nucl. 71(10), 1807-1812 (2008)

33. G. Dattoli, E. Sabia, A. Torre, Nuovo Cimento A 109A, 1425 (1996)

34. H. Goldstein, Classical Mechanics (Addison-Wesley, Cambridge, 1950)

35. H. Minakata, A.Y.U. Smirnov, Phys. Rev. D 70, 073009 (2004)

36. M. Raidal, Phys. Rev. Lett. 93, 161801 (2004) 